\title{
SIFT-BASED SEQUENCE REGISTRATION AND FLOW-BASED CORTICAL VESSEL SEGMENTATION APPLIED TO HIGH RESOLUTION OPTICAL IMAGING DATA
}

\author{
Mickaël Pechaud ${ }^{1,3}$, Ivo Vanzetta ${ }^{2}$, Thomas Deneux ${ }^{3,4}$, Renaud Keriven $^{1}$ \\ ${ }^{1}$ Certis, École des ponts, Marne la Vallée, France \\ ${ }^{2}$ Institut de Neurosciences Cognitives de la Méditerranée, CNRS UMR 6193 \\ Aix-Marseille Université, 13402 Marseille Cedex 20, France \\ ${ }^{3}$ Département d'Informatique, École Normale Supérieure \\ 45 rue d'Ulm, 75005 Paris, France \\ ${ }^{4}$ Department of Neurobiology, Weizmann Institute of Science, Rehovot, Israel
}

\begin{abstract}
Several functional and biomedical imaging techniques rely on determining hemodynamic variables and their changes in large vascular networks. To do so at micro-vascular resolution requires taking into account the - usually small but often non-rigid - mechanical deformations of the imaged vasculature induced by the cardiac pulsation and/or the subjects'body movements. Here, we present two new algorithmic approaches, allowing (i) to efficiently and accurately co-register large sets of such images in a non-rigid manner using Scale-Invariant Feature Transform (SIFT) keypoints, and (ii) to extract blood vessels and their diameters based on blood-flow information using a fast marching algorithm. These methods were applied to optical imaging data of intrinsic signals from awake monkey visual cortex at high spatiotemporal resolution $(30 \mu \mathrm{m}, 5 \mathrm{~ms})$. The movement of red blood cells in the sequences could be enhanced by a Beer-Lambert-based image preprocessing. Our SIFT-based registration could be directly compared to a rigid registration, whereas the vessel extraction algorithm was tested by verifying flow conservation in vascular branching points. Finally, both methods together proved to improve a lot the estimation of the blood velocity in the vessels.
\end{abstract}

Index Terms - blood flow, biomedical imaging, image registration, image enhancement, image segmentation

\section{INTRODUCTION}

The determination of hemodynamic parameters and their changes in extended vascular networks (e.g., cortex, retina) at high spatial resolution is essential for correctly interpreting both functional and biomedical imaging data. Among those parameters, imaging blood flow is crucial for understanding both sensory-induced and pathological hemodynamic responses, as well as for modeling purposes [1]. Moving

All experiments performed according to NIH guidelines red blood cells (RBCs) can be directly "seen" by optical imaging at adequate wavelengths [2], allowing to obtain stationary blood flow values in vascular networks [3]. However, to achieve a robust, fast and reliable determination of the small, eventually neuronal activity-evoked, changes in cerebral blood flow (CBF), some obstacles still have to be overcome [4]. Among those, there is the need for accurate inter-frame alignment of the imaged vascular patterns that move non-rigidly between one frame and another under the effect of mechanical strains. Moreover, the segmentation of the vessels is a highly time-consuming task if relying on user input, but is a challenge for standard automatic methods due to the weakness of contrast of small vessels and ambiguities posed by crossing and branching points.

Here, we present a new algorithmic approach, allowing to efficiently and accurately register images of vasculature subject to non-rigid deformations. Then, instead of relying on the anatomical image we use flow information, enhanced by a Beer-Lambert-based preprocessing, to automatically extract physiologically realistic blood vessels along with their diameter.

\section{METHODS}

\subsection{Sequence Registration}

Images were acquired at $200 \mathrm{~Hz}$ with a CCD, upon illumination at $570 \mathrm{~nm}$, from the primary visual cortex of an awake macaque who had a $1 \mathrm{~cm}^{2}$ transparent cranial window chronically implanted above the area of interest. Even though, during the experiment, the monkey's head is thoroughly stabilized, the curvature of the cortical surface, its position with respect to the camera and the exact morphology of the vasculature change slightly under the effects of the heart-beat and the monkey's body movements. These movements can be as large as a few pixels, and can be relatively fast (until hundred of $\mathrm{Hz}$ ). An interframe "spatial matching" step is thus required to be able to further process each image-sequence. [5] of- 

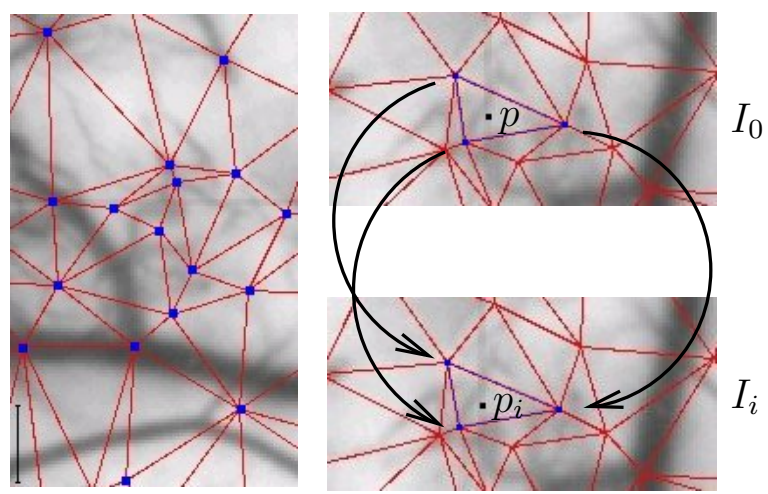

Fig. 1. Left: Blue: SIFT points on a part of the first frame of the sequence. Red: corresponding Delaunay triangulation. Right: Registration between two frames : a point belonging to a triangle in the first image is registered to the point with same barycentric coordinates in the corresponding triangle of the second image.Scalebar is $500 \mu \mathrm{m}$ in all figures

fers a recent survey of several image such image registration methods.

Here, we propose a new features-based method for registering a complete sequence, based on Scale-Invariant Feature Transform (SIFT) keypoints [6]. SIFT is a state-of-the-art fast and robust algorithm for extracting and characterizing salient features from an image which can deal with several computer vision problems. For each image, the SIFT algorithm yields a number (controlled by a threshold) of 2D points $p$ with subpixel precision, along with a descriptor vector $v_{p}$ in $\mathbb{R}^{128}$ for each point $p$, which represents the image around the detected point. The main feature of the SIFT detector is that the points and descriptors obtained are invariant with respect to scale, rotation, and illumination changes.

Our method can be described by the following steps:

1. Features Detection: the SIFT algorithm is applied to each image of the sequence, to detect characteristic points along with their descriptors (fig. 1) after images have been smoothed with a narrow Gaussian filter $(\sim 2$ pixels) to remove high spatial frequency components.

2. Features Matching: we use one frame (usually the first, 0 ) as a reference and match its SIFT keypoints to those of other frames. Using some threshold $\delta$, we keep from the set of these keypoints only those $p_{0}$ which match with one and only one keypoint $p_{i}\left(\left\|v_{p_{0}}, v_{p_{i}}\right\|_{2}<\delta\right)$ in every other frame $i$. Notice that no spatial information is used during this step: only the points' descriptors are used during the matching process, not their positions. This potentially allows for large movements between frames.

3. Full Image Matching: the third step is intended to extend the matching of the characteristic points to the whole space. For this purpose, we first apply Delaunay triangulation [7] to form a mesh $M_{0}$ which vertices are the SIFT points of the reference frame (fig. 1). Then each triangle $\left(p_{0 a}, p_{0 b}, p_{0 c}\right)$ of this mesh is matched to its counterpart $\left(p_{i a}, p_{i b}, p_{i c}\right)$ in each other image $i$ using an affine transformation (figure 1).

\subsection{Flow-based vessels extraction}

\subsubsection{Beer-Lambert correction}

The Beer-Lambert law predicts the measured signals as a function of the absorption of the illumination light by the tissues. If we separate the absorption by the RBCs from the one from vessels or other cortical tissues, we get $I \approx I_{0} e^{-\alpha 2 d} e^{-\beta 2 d^{\prime}}$, where $I$ is the reflected light intensity, $I_{0}$ the incident light, $\alpha$ the absorption coefficient of vessel, $d$ the width of the vessel at the considered point, $\beta$ the absorption coefficient of the RBCs and $d^{\prime}$ its width.

Thus the signal of interest - e.g. the presence of RBCs can be extracted by applying the following filter to each point of the sequence : $d^{\prime} \backsim-\log \left(\frac{I}{I_{\text {base }}}\right) \quad$ where $I_{\text {base }}=I_{0} e^{-\alpha 2 d}$

For each point $I_{b a s e}$ is evaluated as a robust minimal intensity throughout the sequence. Such a normalization using the minimal intensity instead of average intensity [4] enhances the signal from RBCs motion in the vessels without increasing the noise outside.

\subsubsection{Shortest paths}

Our approach is based on shortest paths methods. These methods are widely used for segmenting vessels from anatomical 2D or 3D images [8]. They rely on the fact that in many modalities, gray level is a relevant indicator of the presence of a vessel at any point of the image. For example, in optical imaging data, vessels appear darker than the background. We cast the problem of segmenting a vessel between two usersupplied points $p_{0}$ and $p_{1}$ of an image $I:[0,1]^{2} \rightarrow[0,1]$, into the minimization of the following energy:

$$
E(C)=\int_{0}^{1} f(I(C(t))) d \sigma(t)
$$

where $C:[0,1] \rightarrow[0,1]^{2}$ is a curve such that $C(0)=p_{0}$ and $C(1)=p_{1}, f$ a positive monotonous mapping, and $\sigma(t)$ the Euclidean arc-length. $f(I(C(t)))$ thus drives the curve toward low intensity regions.

Such energy can be globally minimized in any dimension in a very efficient way by using Fast Marching Methods (FMM)[9]. Actually, the problem boils down to finding the shortest path between two points with respect to the metric $[f(I())]. d \sigma$.

\subsubsection{Blood-flow based image segmentation}

In this section, we propose a completely new approach to perform a semi-automatic extraction of vessels based on the 


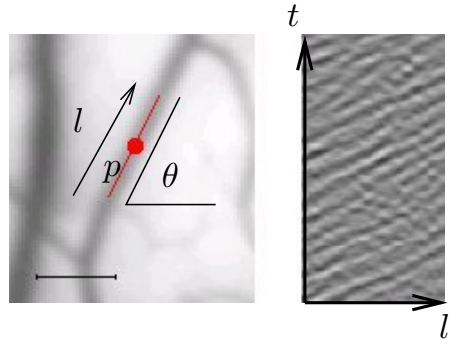

Fig. 2. Extraction of the space - time image. Left: neighborhood of $p$ in the direction $\theta$. Right: corresponding space - time image.

flow information.

To adapt the shortest path formalism to a flow-based extraction of vessels, we replace the light-intensity (gray level) information by a value depending on the presence - or absence - of blood-flow. For a point $p$ and an orientation $\theta$, we determine whether flow following the direction $\theta$ is present at $p$ throughout the sequence. To achieve this, we first extract a 2-dimensional space - time image from a small neighborhood of $p$ in our sequence of frames in direction $\theta$ (Fig. 2), yielding an image $I(l, t)$. Using the same structure tensor formalism as in [4], we compute the following tensor:

$$
T(p, \theta, t)=\left\langle\left(\frac{\partial I}{\partial t}, \frac{\partial I}{\partial l}\right)\left(\frac{\partial I}{\partial t}, \frac{\partial I}{\partial l}\right)^{T}\right\rangle
$$

Let $\bar{T}(p, \theta)$ be its mean over $I(l, t)$. The ratio $\rho(p, \theta)$ between the two eigenvalues of $\bar{T}(p, \theta)$ (i.e. its anisotropy) gives an indicator of the presence of flow in that direction - the more the tensor is anisotropic, the more likely there is significant flow.

Again, given two points $p_{0}$ and $p_{1}$, we consider the space+direction domain $[0,1]^{2} \times[0, \pi)$ (where the direction component is periodic). Using the FMM, it is straightforward to find the shortest path between the sets $\left\{\left(p_{0}, \theta\right) \mid 0 \leq \theta<\pi\right\}$ and $\left\{\left(p_{1}, \theta\right) \mid 0 \leq \theta<\pi\right\}$ with respect to a metric based on the potential $(-\rho)$. Keeping only the spatial component of this path, we obtain an optimal path such that there is significant flow information along it and for which the flow direction changes smoothly.

\subsubsection{Radius extraction}

Going one step further, we now propose a method to evaluate at the same time the radius of the segmented vessel. Briefly, for a point in the space+direction domain, and for any candidate radius $r$, we evaluate the presence of flow in the $\theta$ direction on a neighborhood of radius $r$ perpendicular to the $\theta$ direction. This yields a potential $\rho_{p, \theta, r}$ in the space+direction+radius domain $[0,1]^{2} \times[0, \pi) \times\left[r_{\min }, r_{\max }\right]$. The resulting 4D optimal path is projected back onto the space+radius domain, giving the center and the radius of a tubular structure.

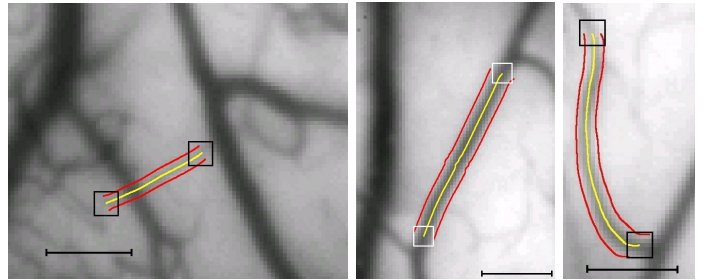

Fig. 3. Automatically extracted vessels. Initial and final points are shown with squares. Notice that only flow information (vs anatomic information) was used to perform these segmentations.
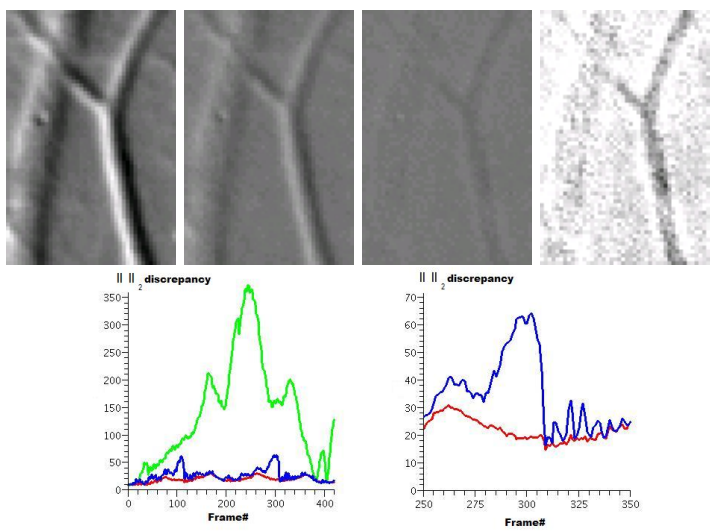

Fig. 4. Top: ratio between frame 0 and frame 300 of a representative sequence, on an area of interest. From left to right : raw (no registration), rigid registration, SIFT-based registration (clipping range i.e. gray-level intensity scale - is the same for the 3 images), SIFTbased registration with a clipping range ten times smaller. Bottom: \|\|$_{2}$ comparison of each frame in the whole sequence to frame 0 (for the area of interest). Raw, rigid registration and SIFT-based registration are respectively represented in green, blue and red. Left: whole sequence. Right: zoom on frames 250 to 350

Fig. 3 shows some results of vessels extracted by this method, superimposed on the first image of the sequence. Note how the smoothness in orientation imposed by our method allows the extraction of the vessel, even when crossings are cluttering the image.

\section{RESULTS}

\subsection{Frame registrations: rigid vs. non-rigid}

Fig. 4 compares the performance of our SIFT-based registration method with a classical rigid registration algorithm. Notice our method correctly registers the borders of the vessels.

\subsection{Average flow in the vasculature}

Fig. 5 shows RBCs' speeds in three automatically segmented vessels. RBCs were found to cross any given section of the vessel one-by-one. Also, linear RBC density along the 


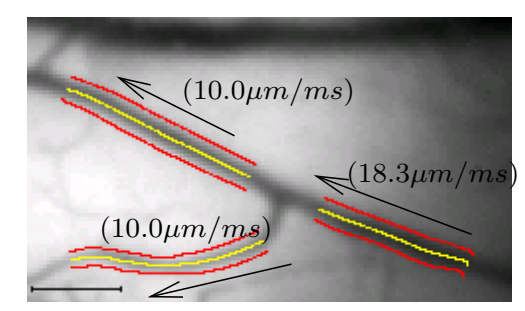

Fig. 5. RBCs' speeds in three automatically segmented vessels
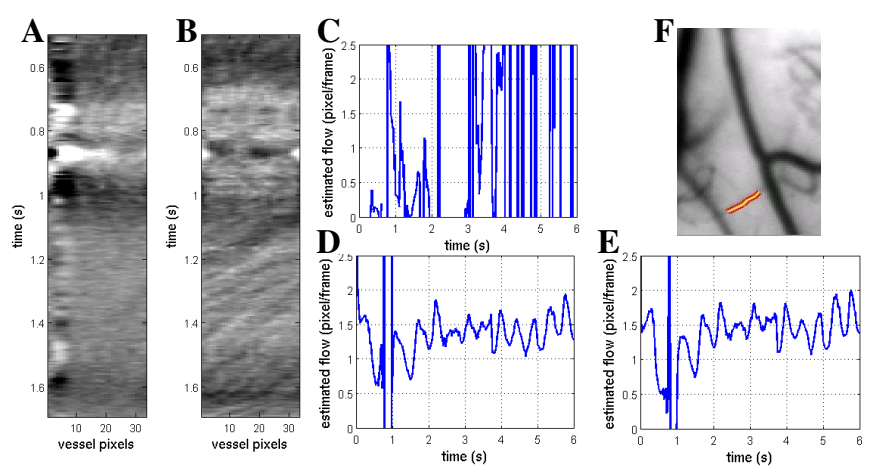

Fig. 6. Comparison of the estimations of RBCs velocity changes after rigid vs non-rigid sequence registration. (F) Vessel considered, extracted using flow-based segmentation. (A,B) Space-time data extracted along this vessel after rigid and non-rigid registrations respectively. (C,D) Corresponding estimates of RBCs velocity, using the tensor structure information: only in the non-rigid case it is possible to estimate the velocity and then detect heart-pulsation changes. (E) Estimation in the non-rigid case, when averaging the structure tensor over the whole section of the vessel: only little information is added compared to using only the middle line of the vessel (D) vessels'axis was found to be essentially equal for all three vessels $\left(D_{1} \sim D_{2} \sim D_{3} \sim 6.7 \pm 1.18 \mathrm{~mm}^{-1}\right)$. The RBC current conservation equation $V_{1} D_{1}+V_{2} D_{2}+V_{3} D_{3}=0$ is thus satisfied within the variability of the data (where $V_{i}$ are the RBCs' speeds in the vessels, and $D_{i}$ the density of RBCs).

\subsection{Variations of the flow in time}

Estimation of velocity changes of the RBCs flow inside the vessels is much sensible to the accuracy of frame registration and vessel extraction. We performed such estimations on a trial of our monkey experiment presenting a high level of vibrations (see [4] for methods). Fig. 6 shows that in the rigid registration case, the data remains too much polluted by signals originating from outside the vessel and no flow estimation is possible; whereas the SIFT registration allows to deal with these vibrations most of the time (except when they are faster than frame acquisition rate, resulting in blurred raw images).

\section{DISCUSSION AND CONCLUSION}

Using the non-rigid image registration described here, we were able to achieve far better spatial matching between the vasculature in different frames (Fig. 5). As a result, the blood flow signal could be recovered in vessels that did not yield any signal upon rigid registration. The obtained RBC flow could also be validated for conservation in vascular branching points, the total number of RBCs flowing in and out being found to match. The described data processing will hopefully allow increasing the accuracy and the sensitivity of optical imaging -based blood flow measurements, in particular with respect to reliably mapping over large vascular networks the small activity dependent blood flow changes elicited by neuronal activation .

\section{REFERENCES}

[1] RB Buxton, K Uludag, DJ Dubowitz, and TT Liu, "Modeling the hemodynamic response to brain activation.," Neuroimage, vol. 23, no. Suppl 1, pp. S220-S233, 2004.

[2] T. Bonhoeffer and A. Grinvald, Brain Mapping: the Methods (Toga, AW, Mazziotta, JC Eds.), chapter Optical imaging based on intrinsic signals: the methodology., pp. 55 - 226 97, Academic Press, California, 1996.

[3] A. Grinvald, T. Bonhoeffer, I. Vanzetta, A. Pollack, E. Aloni, R. Ofri, and D. Nelson, "High-resolution functional optical imaging: from the neocortex to the eye.," Ophthalmol. Clin. North. Am., vol. 17, no. 1, pp. 53-69, 2004.

[4] I. Vanzetta, T. Deneux, G. S. Masson, and O. D. Faugeras, "Cerebral blood flow recorded at high sensitivity in two dimensions using high resolution optical imaging," in ISBI, 2006, pp. 1264-1267.

[5] B. Zitova and J. Flusser, "Image registration methods: a survey," Image and Vision Computing, vol. 21, no. 11, pp. 977-1000, October 2003.

[6] D. Lowe, "Distinctive image features from scaleinvariant keypoints," in International Journal of Computer Vision, 2003, vol. 20, pp. 91-110.

[7] J.-D. Boissonnat and M. Yvinec, Algorithmic Geometry, chapter Voronoi diagrams: Euclidian metric, Delaunay complexes, pp. 435-443, Cambridge University Press, 1998.

[8] L.D. Cohen, "Minimal paths and fast marching methods for image analysis," in MMCV05, 2005, pp. xx-yy.

[9] J. A. Sethian, "Fast marching methods," SIAM Review, vol. 41, no. 2, pp. 199-235, 1999. 